\title{
TOWARDS SELF-CONSISTENT SPECTRAL MODELS FOR OUTBURSTING DISCS IN AM CVn BINARIES
}

\author{
N. Nunn, ${ }^{1}$ D. T. Wickramasinghe, ${ }^{1}$ and L. Ferrario ${ }^{1}$
}

Models for AM CVn discs are usually constructed under the assumption of a steady state. Nevertheless, many of these systems have unstable discs. We have constructed models for the spectral evolution of such discs using a disc instability code (Cannizzo 1984) and detailed calculations of the vertical disc structure assuming hydrostatic equilibrium following El-Khoury and Wickramasinghe (2000).

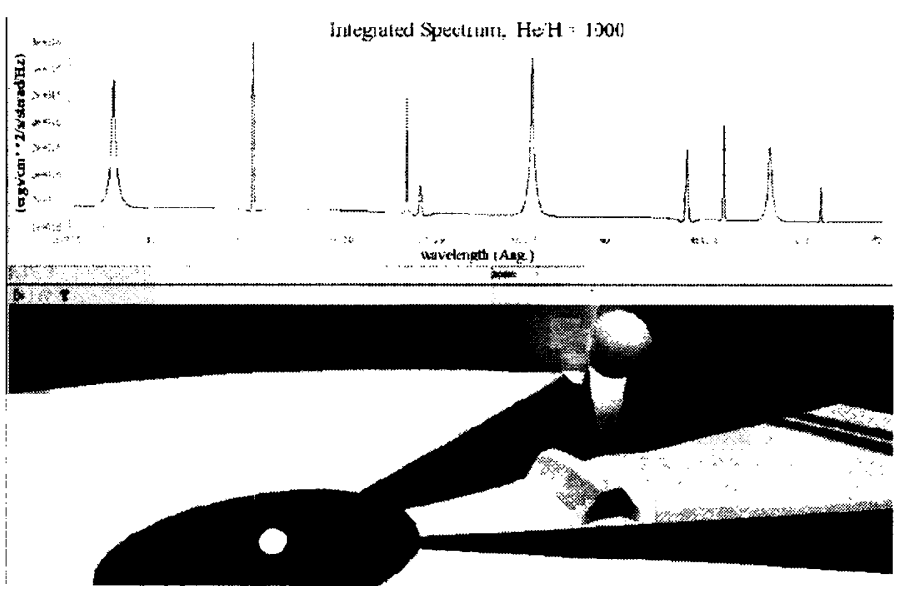

Fig. 1. Low state accretion disc

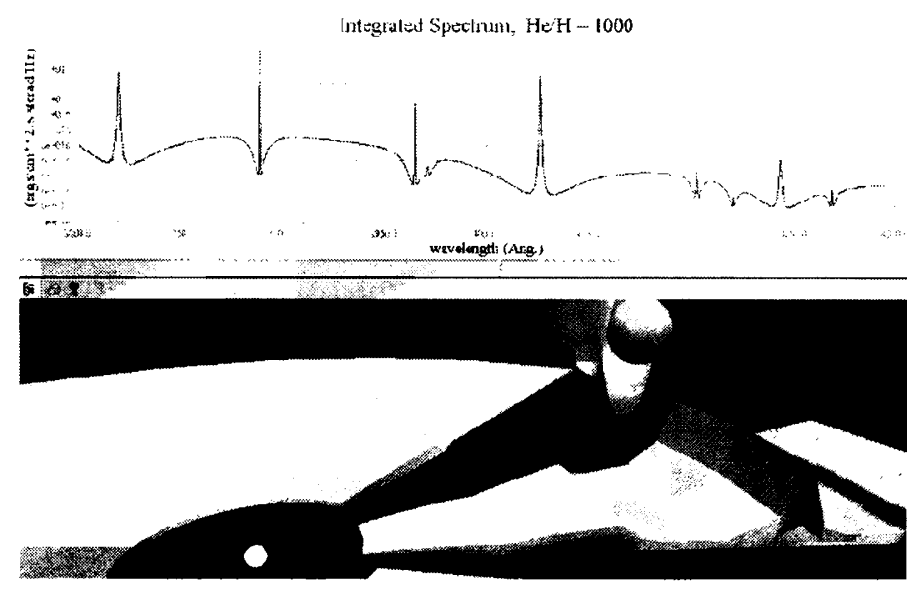

Fig. 2. Transitional state accretion disc

\footnotetext{
${ }^{1}$ Dept. of Mathematics, The Australian National University, Canberra.
}

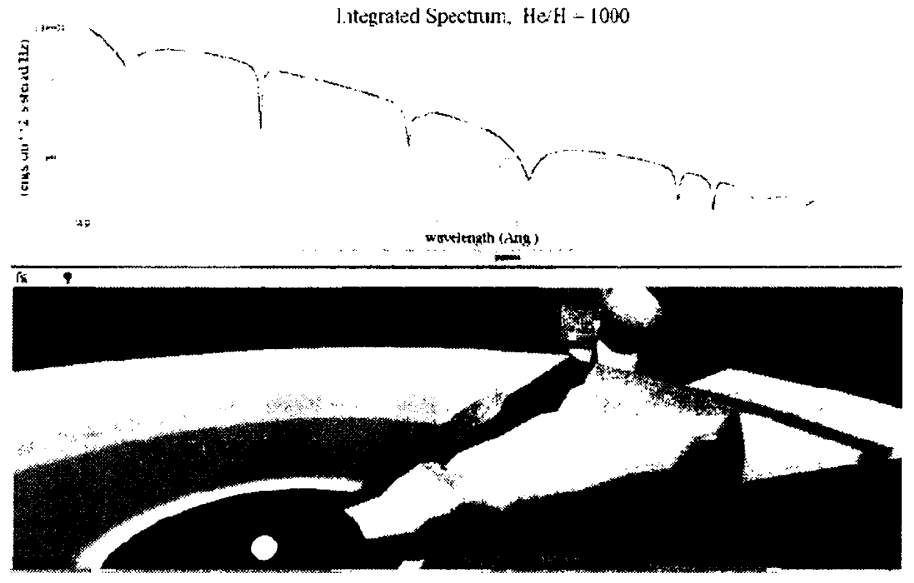

Fig. 3. Outbursting accretion disc

For the present illustration, we consider a binary system of period 10 minutes with a white dwarf primary of mass $M_{2}=0.7 \mathrm{M}_{\odot}$ and a mass losing star of mass $M_{1}=0.066 \mathrm{M}_{\odot}$ - parameters which are possibly relevant for the short period AM Her system ES Cet. We show in Figures 1 to 3 snap-shots of the time dependent discs at three phases representing respectively low, transitional and high states of the disc. In each figure, the bottom panel gives the disc structure and the upper panel the integrated spectrum. We note the change in vertical disc structure as the heating front propagates through the disc. The spectrum changes from a pure emission line spectrum to an absorption line spectrum as the disc passes through an outburst cycle which is an observed characteristic of the sub-class of these systems which show unstable discs. It is generally not possible to mimic these spectra by constructing equivalent constant $\dot{I I}$ discs -that is, despite the compactness of the disc, no single annulus can adequately represent the integrated spectrum.

A detailed account of these calculations will be published in a forthcoming paper.

\section{REFERENCES}

Cannizzo, J. K., 1984, Nature, 311, 443

El Khoury, W. \& Wickramasinghe, D. T., 2000, A \& A. 358,154 